\title{
Resíduos de Pesticidas Organoclorados em Alimentos Gordos
}

\author{
- revisão da metodologia analítica
}

II. Processos de purificação

Celeste de Matos Lino ${ }^{\text {a }}$

Maria Irene Noronha da Silveira ${ }^{\text {a }}$

Os autores apresentam uma revisão dos distintos métodos utilizados na purificação dos extractos destinados à análise de resíduos de pesticidas organoclorados em alimentos cujo teor em gordura excede os $2 \%$.

\section{Introduçāo}

Os extractos dos alimentos cujo teor em gordura excede os $2 \%$ requerem, regra geral, uma purificação mais elaborada do que a utilizada para outro tipo de substracto.

Com o objectivo de conseguir extractos suficientemente purificados que evitem problemas de contaminação na instrumentação analítica posteriormente usada para a detecção e quantificação dos resíduos de pesticidas organoclorados provenientes desses extractos, os analistas têm-se preocupado desde sempre e de forma insistente com esta etapa da metodologia analítica que envolve a análise deste tipo de resíduos.

É nesse sentido que se apresenta uma revisão alargada dos métodos usualmente empregues pelos analistas na purificação destes substractos.

\section{Processos de purificaçāo}

O extracto bruto obtido na fase da extracção necessita de ser tratado por forma a permitir a remoção da maior parte de coextractivos como gorduras e esteróis dado que o material oleoso excedente concentrado a um pequeno volume, poderá provocar danos irreversíveis quando injectado numa coluna de cromatografia gasosa. Esses danos podem manifestar-se de distintas formas: degradação dos pesticidas, fraca resolução, baixas recuperações, respostas estranhas e alterações na sensibilidade do DCE [8].

Os processos de purificação encontrados na bibliografia e aplicados à análise de resíduos de pesticidas organoclorados em alimentos gordos são numerosos.

\section{Purificação prévia por precipitação a baixa temperatura}

Alguns analistas recorrem à purificação prévia dos extractos provenientes de alguns substractos possuidores de um elevado conteúdo lipídico. Essa purificação é conduzida por arrefecimento a $3{ }^{\circ} \mathrm{C} / 1 \mathrm{~h}$, de extractos de óleos e gorduras [13], a $-10^{\circ} \mathrm{C}$ em extractos de leite e de tecidos animais [7] ou a $-70{ }^{\circ} \mathrm{C}$ em extractos de óleos vegetais, manteiga e gorduras animais em benzeno-acetona $(1+19)$ [42] com a finalidade de reduzir a concentração inicial da gordura do extracto, sem alterar a concentração dos resíduos de pesticidas, evitando assim uma sobrecarga lipídica da columa de adsorção a utilizar posteriormente.

\section{Partição líquido-líquido}

A partição líquido-líquido deverá permitir uma extracção eficaz dos resíduos de pesticidas organoclorados da fracção lipídica, evitando a extracção simultânea de grandes quantidades de matéria gorda. Assim, o solvente a usar nesta fase deverá apresentar boa solubilidade face aos resíduos a analisar.

Na generalidade dos processos, os investigadores optam pelo par de solventes acetonitrilo-éter de petróleo ficando os resíduos retidos na fase etéra [2] [32] [41] [45] [51] [54] [57]. Alguns analistas recorrem ao n-hexano na partição líquidolíquido de extractos de leite [7] [14] [63], de ovos [18] [69]; outros preferem aplicar o éter de petróleo a extractos de peixe [14]; de Faubert Maunder [13] e Ballschmiter [5] utilizaram DMF.

\section{Partição em coluna}

Autores há que recorrem a um processo simultâneo de extracção e purificação em coluna. Krynitsky e colaboradores [33] optaram por esta metodologia na determinação do dicofol em ovos. A extracção foi efectuada em columa contendo Florisil desactivado a $2.3 \%$ ( $20 \mathrm{~g}$ ) sobre o qual foi colocada a amostra misturada com sulfato de sódio e a eluição efectuada com éter etílico-hexano $(6+94)$.

Langlois e colaboradores [34] colocaram numa coluna Florisil desactivado a $5 \%(25 \mathrm{~g}-20 \times 600 \mathrm{~mm})$ e sobre este uma mistura de amostra (leite ou derivados) com o mesmo adsorvente e eluindo com diferentes volumes de uma mistura eluente cloreto de metileno-éter de petróleo $(20+80)$ conseguiram separar distintos resíduos de organoclorados.

Porter e Burke [53] optaram pela distribuição de óleos e gorduras num suporte inerte como o florisil não activado efectuando a extracção de baixos níveis de resíduos com água-acetonitrilo $(10+90)$.

\footnotetext{
a Laboratório de Bromatologia - Faculdade de Farmácia, Universidade de Coimbra.
} 


\section{Cromatografia de adsorçâo em coluna}

A análise de resíduos de pesticidas organoclorados nunca foi possível sem a purificação dos extractos, após a extracção e a partição, mesmo quando se recorria à cromatografia em papel que requeria uma purificação muito rigorosa para a obtenção de resultados satisfatórios [46].

Nos nossos dias, a utilização da cromatografia em fase gasosa com detecção por captura electrónica e da espectrometria de massa obriga ao mesmo requisito.

Desde sempre a atenção dos investigadores se tem centrado no estudo de vários adsorventes e nas suas capacidades de adsorção e, apesar de se constatar que diferentes adsorventes se podem empregar, os mais utilizados para este grupo de resíduos são os de mais elevada energia superficial como o florisil, a alumina e o gele de silica [49].

\section{Florisil}

Este adsorvente, na sua forma activada, tem ocupado um lugar de destaque na purificação de diferentes tipos de extractos destinados à análise de resíduos de hidrocarbonetos clorados apesar de apresentar alguns inconvenientes, como aliás foi referenciado pelos autores [38].

O estudo agora apresentado evidencia, de novo, a grande capacidade de aplicação do florisil, mesmo a substractos dotados de altas concentrações de material lipídico.

A preferência dos analistas na purificação destes materiais incide sobre este adsorvente utilizado na forma activada [2] [7] [14] [16] [18] [20] [32] [37] [40] [41] [45] [46] [51] [53] [54] [55] [57] [61] [63] [64]. No entanto, Moats [46] [48] apesar de verificar que o Florisil é o melhor adsorvente na purificação da gorudra da manteiga e na separação da gordura animal, ele necessita de uma desactivação a 5\% com água para que se efectue a eluição da dieldrina. Também Langlois e colaboradores [34] o usaram desactivado, na mesma proporção, com idêntica finalidade. Krynotsky e colaboradores [33] utilizaram o Florisil desactivado a 2,3\% para purificar extractos de ovos destinados à determinação de resíduos de dicofol.

Suzuki e colaboradores [64] usaram o Florisil revestido de nitrato de prata na purificação de extractos de carne de porco, carne de vaca e peixe e separação de resíduos de pesticidas clorados de ftalatos. Chiang e colaboradores [10] numa tentativa de purificar tecido adiposo de bovino utilizaram cartuchos Sep-Pak Florisil ( 0,9 g enchimento) conseguindo boas recuperações de aldrina, hexaclorobenzeno, $\mathrm{PCB}$, e $\mathrm{PBB}_{s}$.

Os extractos obtidos a partir destes substractos apresentam uma sobrecarga lipídica razoável para as colunas de adsorvente mesmo após a partição líquido-líquido. Daqui, a necessidade de utilizar colunas de dimensões apropriadas com a quantidade de Florisil e grau de activação adequado à remoção da totalidade das substâncias lipídicas.

Quando se utiliza um adsorvente vários factores inerentes ou exteriores ao adsorvente têm de ser tomados em atenção. Um dos parâmetros a observar é o diâmetro interno das colunas e, apesar da generalidade dos investigadores recorrerem a diâmetros da ordem dos 20 a 25 mm [2] [20] [32] [33] [35] [41] [45] [51] [54] [57] por obterem separações mais nítidas, há os que optam por macrocolunas de $7 \mathrm{~cm}$ [37] e $4 \mathrm{~cm}$ [46] e outros por minicolunas de $10 \mathrm{~mm}$ [16] [9], $15 \mathrm{~mm}$ [14] [63] [64] e 18 mm [11] de diâmetro.

Liao e colaboradores [37] citam entre as vantagens das macrocolunas usadas na análise de compostos orgânicos semivoláties, em tecido adiposo humano e bovino, evitar a abertura de canais ao longo do adsorvente reduzindo a eficácia de retenção e provocando uma má separação entre os lípidos e os compostos a analisar. Mas, a sua utilização onera grandemente a análise dada a quantidade elevada de Florisil que é necessário $(100 \mathrm{~g}) \mathrm{e}$, consequentemente, mais elevada quantidade de solvente é necessário na eluição, para além de que um aumento de diâmetro da coluna apresenta maior tendência para a formação de zonas irregulares originando tailing na eluição [15].

Holden e Marsden [27] referem que minicolunas de $6 \mathrm{~mm}$ de diâmetro interno com enchimento seco efectuam melhores separações lipídicas mas, com colunas de $18 \mathrm{~mm}$, o enchimento seco torna-se problemático dado que ocorre a abertura de canais. Então, torna-se necessário humedecer o adsorvente no sentido de se obterem melhores separações. Claeys e Inman [11] ao optarem por colunas deste diãmetro conseguiram boas separações.

Dentro das microcolunas, as colunas mais largas dão resolução muito mais pobre [68].

Tendo em atenção que os substractos agora estudados apresentam teores relativamente elevados de material lipídico, por vezes, uma simples purificação com adsorvente não é suficiente para a sua remoção total e alguns analistas recorrem a purificações suplementares. Liao e colaboradores [37] recomendam a purificação adicional com uma segunda coluna de Florisil ( $10 \mathrm{~g}$ ) para proceder à eliminação dos lípidos se o seu teor no eluato é excessivo; Porter e Burke [53] também utilizam uma purificação suplementar com Florisil após a partição em coluna, resultando uma melhoria no caso do óleo do milho.

O sistema eluente influencia de forma acentuada a capacidade de ligação lipídica do adsorvente. O sucesso da separação dos distintos grupos de pesticidas organoclorados depende não só do adsorvente e das substâncias adsorvidas mas, igualmente da aplicação do melhor sistema eluente.

São sobretudo os resíduos de pesticidas organoclorados oxigenados (dieldrina, endrina e heptacloro epóxido) que apresentam maiores problemas de eluição. Assim, Mills [45] utilizando E.E.-E.P. $(6+94)$ não conseguiu eluir dieldrina, endrina e outros resíduos. Para os tentar recuperar, a maioria dos investigadores opta pelo recurso a eluentes mais polares: E.E.-E.P. $(15+85)$ [2] [16] [18] [20] [32] [40] [41] [51] [54] [57] [69]; E.E.-E.P. $(20+80)$ [12]; Clor. Met.-E.P. $(20+80)$ [34] [46]. Para provocar efeito similar alguns analistas aumentam o volume de eluente [34] e outros recorrem ao Florisil desactivado para recuperar satisfatoriamente os pesticidas oxigenados do grupo aldrina [46] [70] e o heptacloro epóxido [34] [70].

Stimac [61] conseguiu eluir a endrina e a dieldrina com o E.E.-E.P. $(6+94)$ lavando previamente a coluna de Florisil com a quantidade adequada de éter etílico, tornando assim a análise mais rápida, o que constitui uma vantagem particularmente quando a presença de resíduos na amostra é vestigiária. 


\section{Alumina}

A alumina tem sido utilizada nalguns casos como alternativa ao Florisil devido à instabilidade da actividade deste.

Vários analistas têm efectuado purificações com este adsorvente. de Faubert Maunder e colaboradores [13] e Zell e Ballschmiter [72] utilizaram-na desactivada a 5\% com água na purificação de variados extractos. Ernest e colaboradores [17] e Ballschimiter e colaboradores [5] usaram-na na forma neutra igualmente desactivada a $5 \%$.

Alguns autores referem mesmo a sua maior capacidade de retenção lipídica e, consequentemente a melhor eficácia na separação dos lípidos e dos resíduos a analisar relativamente ao Florisil desactivado a $5 \%$ e ao gele de sílica desactivado a $10 \%$ [11] [27] quando utilizado na forma desactivada a $5 \%$. Mukherjee [50] faz referência a esta sua acção tanto na forma activada como desactivada. Também Wells e Johnstone [68] referenciam esta importante capacidade de acordo com o grau de desactivação e a quantidade de adsorvente chamando ainda a atenção para o grau de resolução de acordo com o fabricante.

Outros investigadores [40] recorrem à alumina não em coluna mas, adicionada previamente a amostras de leite e sementes oleaginosas para adsorver os lípidos. Gillespie e Waters [20] desenvolveram um processo muito similar para gordura de manteiga mas, com maiores quantidades de alumina desactivada a 16-19\% com água.

Lea [37] efectuou a purificação dos lípidos contidos em amostras de sebo de vaca e gordura de frango procedendo a uma saponificação com hidróxido de sódio etanólico, acidificação com ácido sulfúrico diluído e cromatografia com alumina desactivada a $10 \%$ com água.

\section{Gele de sílica}

Apesar de o gele de sílica ser um adsorvente utilizado por alguns envestigadores na análise de resíduos e pesticidas organoclorados, tem manifestado pouca eficácia na remoção de lípidos quando comparado com os dois adsorventes anteriormente mencionados.

Claeys e Inman [11] referem essa menor capacidade de retenção lipídica quer na forma activada quer desactivada a $10 \%$. Também Mukherjee e colaboradores [50] verificaram que colunas do adsorvente usado isoladamente ou em mistura com Celite apresentam uma remoção pouco eficaz de gorduras. Moats [46] refere mesmo que o gele de sílica adsorve fortemente o lindano e os compostos oxigenados do grupo drina como a dieldrina.

Samuel [56] refere que colunas de ácido silícico não se mostram satisfatórias para a purificação e separação dos pesticidas organoclorados quando os extractos se apresentam sujos ou quando a sua quantidade é excessivamente grande.

Wells e Johnstone [68] efectuaram a separação de distintos resíduos de pesticidas organoclorados em quatro grupos recorrendo a duas microcolunas $(6 \mathrm{~mm}$ d.i) geminadas uma com $3 \mathrm{~g}$ de alumina desactivada a $4 \%$ e outra de $2,5 \mathrm{~g}$ de gele de sílica desactivado a $3 \%$ usando dois eluatos de $n$-hexano por coluna. A aplicação desta técnica reduz em cerca de $50 \%$ o tempo de análise para além de permitir uma quantificação adequada dos resíduos em amostras de tecidos animais. Voogt e colaboradores [66] apresentaram conclusões similares com colunas de $200 \times 5 \mathrm{~mm}$.

\section{Carvão}

O carvão é aplicado principalmente na purificação de extractos de plantas destinadas à análise de resíduos de pesticidas organoclorados devido à sua acção eficiente na remoção de pigmentos, ceras e gorduras [38].

O seu emprego na análise de resíduos de pesticidas organoclorados em alimentos com elevado teor em gordura è restrita para além de que algumas das técnicas que surgiram nesse campo exigiam volumes de eluentes demasiado elevados [47] e, alguns dos componentes de baixo peso molecular presentes nalguns extractos (manteiga), não são retidos de forma eficaz em colunas de carvão.

Samuel [56] utilizou o carvão activado misturado com óxido de alumínio, celite, sulfato de sódio e attapulgus clay para purificar diferentes tipos de extractos.

Embora Mukherjee e colaboradores [50] ao efectuarem o estudo da eficiência de várias colunas com diferentes adsorventes tenham verificado que o carvão activo se mostrou mais eficaz na remoção de pigmentos e gorduras, McLeod e colaboradores [44] ao estudarem dez tipos diferentes de carvão verificaram que a sua capacidade de retenção para ceras e óleos não era tão elevada quanto para os pigmentos. Adachi e colaboradores [1] estabeleceram um método para a análise de amostras de leite procedendo à purificação com $0,5 \mathrm{~g}$ de carvão activado contido em minicolunas de $15 \mathrm{~mm}$ de d.i. seguida de eluição com acetona-hexano $(1+1)$ e a extracção dos pesticidas com hexano.

\section{Cromatografia de permeaçāo de gele}

As purificações por cromatografia de permeação de gele baseam-se na exclusão molecular separando as moléculas de lípidos de elevado peso molecular $(\geq 600)$ das moleculas dos pesticidas cujos pesos moleculares oscilam entre 200 e 400 . As moléculas de lípidos apresentam menor tempo de retenção pelo que são eluídas antes das moléculas dos pesticidas. Diferentes tipos de geles têm sido utilizados neste tipo de cromatografia [38]. Stalling e colaboradores [60] ao efectuarem um estudo comparativo com Bio Beads SX-2, SX-4 e SX-8, usando como solventes o tolueno, cloreto de metileno e ciclohexano concluiram que para a separação de lípidos e pesticidas em extractos de peixe, o sistema cromatográfico Bio Beads SX-2-ciclohexano mostrou maior eficácia. Os autores verificaram igualmente que o Sephadex LH-20 se mostrou inadequado para a purificação de lípidos em extractos similares, destinados à cromatografia gasosa. Revelaram também que, comparando com a purificação por partição líquida aliada ao Florisil, a cromatografia de permeação de gele revelou uma recuperação cerca de $7 \%$ mais elevada para o p,p'-DDE.

Baseados nesta técnica, Tindle e Stalling [65] desenvolveram um sistema automatizado conseguindo desta forma para além de uma maior rapidez de execução, uma melhoria de precisão analítica e diminuição de perdas resultante de uma menor manipulação. 
Griffitt e Craun [23] fundamentados nos trabalhos anteriormente mencionados, aplicaram ambas as técnicas (manual e automatizada) a amostras de leite conseguindo que cerca de 98\% de lípidos eluissem antes da fracção que contêm a quase totalidade dos pesticidas.

Posteriormente, Stalling [59] desenvolveu um sistema cromatográfico Bio Beads SX-3 e tolueno-acetato de etilo $(1+3)$ e aplicando-o a uma diversidade de amostras incluindo lípidos provenientes de plantas, extractos de rações, tecido adiposo humano e sebo de boi, conseguiu purificações da ordem dos $1500 \mathrm{mg}$ de lípidos. Johnson e colaboradores [31] confirmaram boas recuperações para uma gama de resíduos de organoclorados e organofosforados contidos em amostras de gorduras animais e óleos vegetais fraccionados pelo mesmo sistema usado por Stalling [59].

Young e Kamps [71] procedendo à análise do óleo de soja crú, seus subprodutos e do óleo completamente refinado por cromatografia de permeação de gele automatizada com Bio Beads SX-3 e tolueno-acetato de etilo $(1+3)$ verificaram que os pesticidas organoclorados não polares se concentram durante o processo de refinação apresentando factores de concentração da ordem dos 100 .

Specht e Tillkes [58] aplicaram Bio Beads SX-3 com o sistema solvente acetato de etilo-ciclohexano $(1+1)$ à separação de resíduos de pesticidas organoclorados e organofosforados contidos em alimentos de origem animal e vegetal.

Hopper [28] ao utilizar ORPVA-2000 com cloreto de metileno-acetona $(30+70)$ para a remoção dos lípidos em cinco tipos de gordura conseguiu melhores recuperaçōes relativamente ao sistema anteriormente experimentado pela FDA, Bio Beads SX-2-cloreto de metileno-ciclohexano $(10+90)$.

Aquele sistema permite menores volumes de eluição (máx. $130 \mathrm{ml}$ ), facilidade de evaporação em concentrador Kuderna-Danish, para além de que o sistema automatizado permite determinar uma gama variada de compostos orgânicos incluindo pesticidas organoclorados, organofosforados, herbicidas do ácido clorofenóxi, carbamatos e outros produtos químicos.

O mesmo autor [29] desenvolveu o sistema BioBeads SX-3 e cloreto de metileno-n-hexano (50:50) para purificação de diferentes substractos ricos em gordura. Este sistema apresenta melhorias relativamente aos outros sistemas já desenvolvidos que empregam o mesmo gele: diminuição do solvente de eluição $(60-140 \mathrm{ml})$, maior facilidade de evaporação, uma fracção «dump» mais pequena $(0-60 \mathrm{ml})$ contendo $99 \%$ de gordura para recuperações idênticas de pesticidas organoclorados e organofosforados.

Ault e Spurgeon [4] desenvolveram um processo automatizado com Bio Beads SX-3 e cloreto de metileno-ciclohexano em proporções iguais que foi adoptado como método oficial na purificação de gorduras de aves e resíduos de hidrocarbonetos clorados não iónicos.

Com vista a determinar o grau de contaminação com organoclorados de tecido adiposo humano, Lebel e Williams [36] procederam ao fraccionamento dos resíduos e da gordura com o sistema Bio Beads SX-3 (60 g) e cloreto de metilenociclohexano $(1+1)$. Após purificação adicional com Florisil, os extractos foram analisados por cromatografia gasosa com colunas capilares de diferentes polaridades e a confirmação dos resultados por cormatografia gasosa-espectrometria de massa.

Hopper e Grifft [30] aliaram a cromatografia de permeação de gele automatizada a um sistema de colheita e evaporação contínua do efluente da coluna de cromatografia de permeação de gele dissolvendo o resíduo num volume fixo de solvente. As perdas dos resíduos são minimizados por adição de parafina a $0,2 \%$ em cloreto de metileno.

Em muitos casos os eluatos procedentes da cromatografia de permeação de gele têm que ser submetidos a uma purificação suplementar, normalmente efectuada em coluna de Florisil. Quando é necessário proceder à confirmação dos resultados por cromatografia em camada fina ou por cromatografia gasosa-espectrometria de massa, os eluatos devem possuir uma elevada limpidez pelo que têm de ser submetidos a uma segunda purificação com Florisil [23] [36].

Por outro lado, alguns tipos de substractos como os subprodutos da refinação do óleo de soja [31] [71], destilados de plantas contendo material lipídico [31] necessitam igualmente desta purificação adicional. A determinação dos ésteres do grupo clorofonóxi necessita também desta segunda purificação [31].

Para separação dos resíduos de pesticidas organoclorados dos $\mathrm{PCB}$ recorre-se normalmente a uma purificação com minicoluna $7,5 \times 230 \mathrm{~mm}$ de gele de sílica desactivado a 1,5\% com água [58].

\section{Sweep co-distillation}

Esta técnica de purificação consiste em injectar o extracto numa corrente contínua de azoto alcançando um tubo de vidro aquecido (tubo de Storherr) que contém lã de vidro, pérolas de vidro ou Anakrom e areia. Neste tubo impurezas várias incluindo lípidos e os resíduos de pesticidas permanecem depositados. Co-destilando éter de petróleo ou outro solvente, os resíduos de pesticidas são condensados num banho de água e gelo e, posterirmente transferidos para a coluna de Florisil.

Storherr e colaboradores [62] adaptaram esta técnica, inicialmente por eles desenvolvida e aplicada à análise de resíduos de organofosforados em frutos e vegetais, a 14 hidrocarbonetos clorados em óleos comestíveis encontrando boas recuperações. No entanto, para a quantificação dos resíduos por CG-DCE é necessário o recurso à purificação suplementar em microcoluna de florisil. Esta técnica apresenta-se rápida, simples, directa, fácil de realizar e utiliza quantidades mínimas de solventes.

Watts e Storherr [67] utilizaram esta purificação em extractos de leite com vista à determinação de resíduos de pesticidas organoclorados e organofosforados utilizando Anakrom para enchimento do tubo de Storherr, operando a temperaturas de $180 \pm 2{ }^{\circ} \mathrm{C}$. As recuperaçōes obtidas foram de $\cong 90 \%$.

Esta técnica foi aplicada a outros alimentos de origem animal por Goeke [22].

$\mathrm{O}$ autor encontrou recuperações para os resíduos de pesticidas organoclorados oscilando entre $70 \%$ para o metoxicloro e $101 \%$ para o HCB. Nalguns casos mais de $10 \%$ de DDT é convertido, no tubo de Storherr, em tetraclorodifeniletano. Luke e colaboradores [39] utilizaram o sistema Unitrex que 
permite o tratamento simultâneo de 10 amostras. O tubo de fraccionamento $\left(\Delta 235^{\circ} \mathrm{C}\right)$ é desenhado por forma a que a amostra passe para um tubo central com azoto $(230 \mathrm{ml} / \mathrm{min})$ e depois sobe e desce nas pérolas de vidro sinalizados que estão colocadas no espaço entre o tubo central e as paredes de vidro. Os pesticidas são recolhidos num tubo lateral que contém sulfato de sódio e Florisil desactivado a $1 \%$. A eluição é efectuada com hexano - éter etílico $(90+10)$. Os autores recorrem a este sistema na purificação da gordura de vaca. As recuperações obtidas oscilam entre $83 \%$ para o lindano e $105 \%$ para o p,p'-DDD.

Este sistema permite uma redução dos custos, dado que a amostra utiliza apenas $12 \mathrm{ml}$ de solvente, 6,91 de azoto e menos que $1 \mathrm{~g}$ de Florisil podendo este ser usado novamente, após recuperação. Podem ser purificadas cerca de 30 amostras por dia.

\section{Destilação assistida}

A destilação assistida é apenas uma melhoria da sweep codistilattion em que se elimina o solvente que co-destila no tubo de destilação. Foi aplicada à análise de gorduras animais por Health e Black [24]. Posteriormente, estes analistas [25] introduziram alterações à sua técnica original. Essas modificações constam de um tubo condensador em forma de U mais longo contendo pérolas de vidro de $0,5 \mathrm{~mm}$. Apesar da elevada eficiência deste condensador ele necessita de um banho de gelo e, normalmente, é necessária uma purificação suplementar com Florisil antes da quantificação dos resíduos de organoclorados por CG-DCE.

McDougall [43] descreve um tubo de retenção (100 mmx14 $\mathrm{mm}-65 \mathrm{~mm}-4,5 \mathrm{~mm} ; \Delta 230^{\circ} ; \mathrm{N} 2-600 \mathrm{ml} / \mathrm{min}$ ) para gorduras cujo enchimento é efectuado com Florisil. Este sistema simples e de fácil manejo evita o recurso ao arrefecimento. As análises efectuadas com óleo de manteiga e gordura de boi, com vista à determinação de resíduos de pesticidas organoclorados, conduziram a recuperações de $80 \pm 2 \%$ para o p,p' DDT e $102 \%$ para a dieldrina.

Novas alterações foram introduzidas por Health e Black [26] utilizando um tubo de destilação assistida que não contêm nem pérolas de vidro nem lã de vidro. A purificação dos pesticidas efectua-se em dispositivo contendo Florisil parcialmente desactivado a $5 \%$ com água, semelhante ao de McDougall [43]. A resolução do tubo mostrou-se idêntica à do tubo Unitrex anteriormente desenvolvido por Luke e colaboradores [39]. Operando a uma temperatura de $230^{\circ} \mathrm{C}$ e com um fluxo de azoto de $250 \mathrm{ml} / \mathrm{min}$, as recuperações obtidas dos pesticidas organoclorados em amostras de manteiga e gordura de gado bovino variam de $87 \%$ para o DDT e $104 \%$ para o heptacloro epóxido.

\section{Outros métodos}

Alguns investigadores suspendem a gordura sobre partí-culas de silicato de cálcio sintético, promovendo posteriormente a remoção dos pesticidas organoclorados com mis-tura ace-tona-acetonitrilo $(5+95)$ [55]; outros analistas preferem o estearato de cálcio para promoverem a coagulação dos materiais lipídicos [52].

A cromatografia líquida de alta resolução semi-preparativa também tem sido usada como processo de purificação.
Gillespie e Walters [21] procederam à separação dos pesticidas e $\mathrm{PCB}_{\mathrm{s}}$ de $500 \mathrm{mg}$ de gordura de manteiga com uma coluna de sílica (Zorbax Sil-9,4 mm d.i.x25 cm).

\section{Referências}

[1] Adachi, K. et al .«J. Assoc. Off. Anal. Chem.», 66 (6), 1983, p. 1315-1318. [2] Al-Omar, M.A. et al. «Bull. Environ. Contam. Toxicol.», 36, 1986, p. 109. -113 .

[3] Ang, C.Y.W.; Dugan, L.R. «J. Assoc. Off. Anal. Chem.», 56 (3), 1973, p. 718-720.

[4] Ault, J.A.; Spurgeon, T.E. «J. Assoc. Off. Anal. Chem.» 67 (2), 1984, p. 284-289.

[5] Ballschmiter, K. et al. «Fresenius Z. Anal. Chem.», 306, 1981, p. 323-339 [6] Beroza, M.; Bowman, M.C. «J. Assoc. Off. Anal. Chem.», 49 (5), 1966, p. 1007-1012.

[7] Braun, H.E.; Lobb, B.T. «Can. J. Anim. Sci.», 56, 1976, p. 373-376. [8] Burke, J.A. «J. Assoc. Off. Anal. Chem.», 48 (5), 1965, p. 1037-1058. [9] Bush, B. et al. «J. Assoc. Off. Anal. Chem.», 66 (2), 1983, p. 248-255. [10] Chiang, T.C.H. et al. «J. Assoc. Off. Anal. Chem.», 70 (1), 1987, p. 100-102 .

[11] Claeys, R.R.; Inman, R.D. «J. Assoc. Off. Anal. Chem.», 57 (2), 1974, p. 399-404.

[12] Davidson, A.W. «J. Assoc. Off. Anal. Chem.» 49 (2), 1966, p. 468-472. [13] de Faubert Maunder, M.J. et al. «Analyst», 89, 1964, p. 168-174.

[14] Dogheim, S.M. et al. «J. Assoc. Off. Anal. Chem.», 71 (5), 1988, p. 872 -874 .

[15] Drake, B. «Anal. Chim. Acta», 3, 1949, p. 452-467.

[16] Erney, R.D. «J. Assoc. Off. Anal. Chem.», 57 (3), 1974, p. 576-579.

[17] Ernst, W. et al, «Fresenius Z. Anal. Chem.», 272, 1974, p. 358-363.

[18] Finsterwalder, C.E. «J. Assoc. Off. Anal. Chem.», 59 (1), 1976, p. $169-$ -171 .

[19] Gabica, J. et al. «J. Assoc. Off. Anal. Chem.», 57 (1), 1974, p. 173-175. [20] Gillespie, A.M.; Walters, S.M. «J. Assoc. Off. Anal. Chem.», 67 (2), 1984 , p. 290-294.

[21] Gillespie, A.M.; Walters, S.M. «J. Liq, Chrom.», 9 (10), 1986, p. 2111 -2114 .

[22] Goeke, G. «Dtsch. Lebensm.-Rundsch.», 71 (9), 1975, p. 309-314 in Pest. Abst., 9 (1), 1976, 76-0285.

[23] Griffitt, K.R.; Craun, J.C. «J. Assoc. Off. Anal. Chem.», 57 (1), 1974, p. 168-172.

[24] Health, A.B.; Black, R.R. «J. Assoc. Off. Anal. Chem.», 62 (3), 1979, p. 757-763.

[25] Health, A.B.; Black, R.R. «J. Assoc. Off. Anal. Chem.», 63 (2), 1980, p. 529-531.

[26] Health, A.B.; Black, R.R. «J. Assoc. Off. Anal. Chem.», 70 (5), 1987, p. 862-865.

[27] Holden, A.V.; Marsden, K. «J. Chrom.» 44, 1969, p. 481-492.

[28] Hopper, M.L. «J. Assoc. Off. Anal. Chem.» 64 (3), 1981, p. 720-723.

[29] Hopper, M.L. «J. Agric. Food. Chem.», 30 (6), 1982, p. 1038-1041.

[30] Hopper, M.L.; Griffitt, K.R. «J. Assoc. Off. Anal. Chem.», 70 (4), 1987, p. $724-726$.

[31] Johnson, L.D. et al. «J. Assoc. Off. Anal. Chem.», 59 (1), 1976, p. $174-$ $-187$.

[32] Johnson, L.Y. «J. Assoc. Off. Anal. Chem.", 48 (3), 1965, p. 668-675. [33] Krynitsky, A.J. et al. «J. Assoc. Off. Anal. Chem.», 71 (3), 1988, p. 539 -542 .

[34] Langlois, B.E. et al. «J. Agric. Food Chem.», 12 (3), 1964, p. 243-245. [35] Lea, R. «J. Assoc. Off. Anal. Chem.» 59 (1), 1976, p. 224-225.

[36] Lebel, G.L.; Williams, D.T. «J. Assoc. Off. Anal. Chem.» 69 (3), 1986, p. $451-458$.

[37] Liao, W. et al. «J. Assoc. Off. Anal. Chem.», 71 (4), 1988, p. 742-747. [38] Lino, C.M.; Silveira, M.I.N. «Bol. Fac. Farm. Coimbra», 1987, em publicação.

[39] Luke, B.G. et al. «J. Assoc. Off. Anal. Chem.» 67 (2), 1984, p. 295-298. [40] Luke, M.A.; Doose, G.M. «Bull. Environ. Contam. Toxicol.», 32, 1984, p. $651-656$.

[41] Matsumoto, H. et al. «Bull. Environ. Contam. Toxicol.», 38, 1987, p. 954-958.

[42] McCully, K.A.; Mckinley, W.P. «J. Assoc. Off. Anal. Chem.», 47 (4), 1964 , p. $652-659$.

[43] McDougall, K.W. «J. Assoc. Off. Anal. Chem.», 63 (6), 1980, p. 1355-1356 .

[44] McLeod, H. A. et al. «J. Assoc. Off. Anal. Chem.», 50 (6), 1967, p. 1216-1228 . 
[45] Mills, P.A. «J. Assoc. Off. Anal. Chem.», 42 (4), 1959, p. 734-740.

[46] Moats, W.A. «J. Assoc. Off. Anal. Chem.», 46 (2), 1963, p. 172-176.

[47] Moats, W.A. «J. Assoc. Off. Anal. Chem.», 47 (3), 1964, p. 587-591.

[48] Moats, W.A. «J. Assoc. Off. Anal. Chem.», 49 (6), 1966, p. 973-975.

[49] Morley, H. V. «Res. Rev.» 16, 1966, p. 1-29.

[50] Mukherjee, G. et al. «J. Food. Sci. Tecnol.», 11 (4), 1974, p. 175-176 in Pest. Abst., 9 (2), 1976, p. 132, 76-0568.

[51] Official Methods of Analysis of the AOAC, Washington, D.C. 14th Ed., 1984 , p. $537-538$.

[52] Onley, J.H.; Bertuzzi, P.F. «J. Assoc. Off. Anal. Chem.», 49 (2), 1966, p. 370-374.

[53] Porter, M.L.; Burke, J.A. «J. Assoc. Off. Anal. Chem.», 56 (3), 1973, p. 733-738.

[54] Porter, M.L. et al. «J. Assoc. Off. Anal. Chem.», 53 (6), 1970, p. 1300$-1303$.

[55] Rogers, W.M. «J. Assoc. Off. Anal. Chem.», 55 (5), 1972, p. 1053-1057.

[56] Samuel, B.L. «J. Assoc. Off. Anal. Chem.», 49 (2), 1966, p. 346-353.

[57] Sawyer, L.D. «J. Assoc. Off. Anal. Chem.», 65 (5), 1982, p. 1122-1128.

[58] Specht, W.; Tillkes, M. «Fresenius Z. Anal. Chem.», 301, 1980, p. 300307.

[59] Stalling, D.L. «3rd International Conference on Pesticide Chemistry», 1974, July 1-9, Helsinki-Finland in «J. Assoc. Off. Anal. Chem.», 59 (1), 1976, p. 174-187.

[60] Stalling, D.L. et al. "J. Assoc. Off. Anal. Chem.», 55 (1), 1972, p. 32-38.

[61] Stimac, R.M. «J. Assoc. Off. Anal. Chem.», 62 (1), 1979, p. 85-88.

[62] Storherr, R.W. et al. «J. Assoc. Off. Anal. Chem.», 50 (3), 1967, p. 605-615 .

[63] Suzuki, T. et al. «J. Assoc. Off. Anal. Chem.», 62 (3), 1979, p. 681-684

[64] Suzuki, T. et al. «J. Assoc. Off. Anal. Chem.», 62 (3), 1979, p. 689-694.

[65] Tindle, R.C.; Stalling, D.L. «Anal. Chem.», 44 (11), 1972, p. 1768-1773.
[66] Voogt, P. et al. «J. Chrom.», 363, 1986, p. 407-411.

[67] Watts, R.R.; Storherr, R.W. «J. Assoc. Off. Anal. Chem.» 50 (3), 1967, p. 581-585.

[68] Wells, D.E.; Johnstone, S.J. «J. Chrom», 140, 1977, p. 17-28.

[69] Wessel, J.R. «J. Assoc. Off. Anal. Chem.», 52 (1), 1969, p. 172-175.

[70] Wood, B.J. «J. Assoc. Off. Anal. Chem.», 49 (2), 1966, p. 472-473.

[71] Young, S.J.V.; Kamps, L.R. «J. Assoc. Off. Anal. Chem.» 65 (4), 1982, p. 916-920.

[72] Zell, M.; Ballschmiter, K. «Fresen. Z. Anal. Chem.», 300, 1980, p. 387 -402 .

Abreviaturas:

ACN - Acetonitrilo

ACT - acetona

AC. Et. - acetato-etilo

Benz. - benzeno

Clor. Met. - cloreto metileno

CG. - cromatografia gasosa

DCE - detector captura electrónica

DMF - dimetilformamida

E.E. - éter etílico

E.P. - éter petróleo

FDA - Food and Drug Administration

Hex. - hexano

HCB - hexaclorobenzeno

$\mathrm{PCB}_{\text {s }}$ - bifenilos policlorados

$\mathrm{PBB}_{\mathrm{s}}$ - bifenilos polibromados

Sulf. Sódio - sulfato de sódio

\section{A SPQ é sua amiga \\ Você é amigo dos seus amigos Os seus amigos são amigos da SPQ?}

\section{BOLETIM DE INSCRIÇÃO SPQ}

Nome

Morada

Telefone

Delegação a que deseja pertencer: Coimbra

Lisboa

Porto

Endereço Profissional

Habilitações Académicas

Cargo que desempenha

Junto envio o cheque $n^{\circ}$

Banco

referente à minha quota da SPQ no ano de 1990.

de de 1990

Assinatura 